\title{
Annotation to the Mathematics Connotation in the Perspective of Philosophy and Education
}

\author{
Feng Tian \\ School of Mathematics and Information Science, Shaanxi Normal University, Xi'an, China \\ fengt@snnu.edu.cn
}

Keywords: Philosophy; Education; Educational philosophy; View

\begin{abstract}
Mathematics connotation could be annotated in the different perspective. Different person has different view. In the point of mathematical education, philosophy and education are both very important fields. The field of philosophy lays particular emphasis on the original source and the nature of mathematics. The field of education lays particular emphasis on the comprehension of educational mathematics and pedagogical mathematics content knowledge. The field of educational philosophy lays emphasis of the general educational purpose and values. Combination of both could be formed a new view to portray the philosophy of mathematics education.
\end{abstract}

\section{从哲学与教育的视角诠释数学的内涵}

田枫

陕西师范大学 数学与信息科学学院, 中国 陕西 西安, 710119

fengt@snnu.edu.cn

摘要: 数学内涵可以从不同的视角加以诠释, 角度不同, 内涵迥异。哲学和教育是两个十分 重要的视角, 哲学统领一切学科, 从哲学的视角侧重于思考数学的本源以及数学的学科性质; 教育传承一切文化, 从教育的视角侧重于理解数学教育的发展以及数学教学的合理定位。将 两者的结合, 从哲学与教育的视角诠释数学的内容, 落脚于刻画数学教育哲学的一种来源。

关键词: 哲学; 教育; 教育哲学; 视角; 数学

1. 引言

在各类文献中, 关于数学的描述有很多, 角度不同, 内容迥异。 $\mathrm{R}$-柯朗和 $\mathrm{H}$ - 罗宾在其著作 《什么是数学》 [1] 中从数学家的视角对整个数学领域中的思想和方法做了精辟而生动的阐 述, 但数学的内涵究竟是什么呢, 没有明确。一些数学家、科学家, 乃至哲学家对数学的认 识也有独特的视角, 如培根的 “数学是思维的体操” ; 爱因斯坦的 “数学是思想王国的音乐”; $\mathrm{M}$ - 克莱因的 “数学是一种理性精神” ; 以及 “数学在本质上是具有精确含义的一门语言” 等 [2]，这些观点侧重于对数学某一属性的凸显，但不是对数学内涵的刻画。

迄今为止, 在对数学内涵的刻画中, 被索引频率最高的当属恩格斯的 “数学是研究现实世界 的数量关系和空间形式的科学” , 在当代的字典 [3-4]和我国教育部新一轮修订的课程标准中 都是这样界定的。另一个得到了多数数学家认同的，是 20 世纪 30 年代由法国布尔巴基学派 提出的: 数学是研究模式和秩序的科学。从数学本身的发展来看, 数学的研究对象在不断地 拓展, 从单纯的数与形, 到数形结合, 到可能的量的关系和形式, 到既不是数量关系也不是 空间形式 [5]。也就是说, 数学研究对象的发展已经从对现实世界的直接抽象与概括, 拓展为 人类思维的一种能动创造。随着时代的发展, 数学日益成为公民素养的重要组成部分, 本文 试图分别从哲学和教育两个视角对数学的内涵进行诠释, 并最终落脚于刻画数学教育哲学的 一种来源。 


\section{2. 从哲学的视角对数学内涵的诠释}

\section{1. 哲学的视角}

哲学是关于世界观的学问, 是关于自然、社会和思维普遍规律的整体认识的理论, 是本体论、 认识论和价值论的统一的思想体系。哲学是自然科学、社会科学以及思维科学的概括和总结, 是从各个具体学科中概括、总结出来的最一般的知识 [6]。人类的一切认识都离不开哲学, 尽 管对于绝大多数人来说并不知道哲学究竟是什么, 但每个人都是自发的哲学家, 每一种认识 都遵循了一定的哲学思想。

2. 2. 诠释的角度

以数学为例, 在学习的过程中, 会出现类似于 “为什么负负得正”, “为什么逢十进一”, “反证法证明的结论一定正确吗”，“虚数是一个真实的数吗” 等问题; 甚至还有更深一层 的思考, 如 “数学到底是什么, 是独立于人的思维客观存在的物质, 冥冥之中已有定数, 只 是假以数学家之手找出的固有规律; 还是思想纯粹建构的产物, 是数学家们的创造、发 明” ……, 这些最朴实的问题不仅仅是数学问题, 也是哲学问题, 是关于数学如何发生、发 展的元数学问题, 蕴含于数学认识论、数学观之中, 是一种从哲学视角对数学的思考与诠释。 这种诠释可以从两个角度展开, 一是数学的本源性思考, 二是数学的学科归属。首先, 人类 对数学的本源性思考经历了以下历程。最初, 古希腊先哲柏拉图的客观唯心主义先验论认为 数学是纯粹的心灵创造物。对于这些古希腊先哲而言, 数学构成了代表宇宙真实存在的、独 立的、永恒的理念世界的一部分, 它依赖于时空和人的思维才永恒存在, 毕达哥拉斯的名言 “万物皆数” , 开普勒的三大定律, 门捷列夫的化学元素周期表, 都表明冥冥宇宙, 世间万 物皆有定数的人类认识。在十九世纪, 随着数学第三次数学危机, 现代数学基础理论产生出 关于数学和逻辑关系的不同认识的三大派别: 逻辑主义、直觉主义和形式主义, 各个学派对 数学的诠释出现了不同的观点。

\section{3. 数学的内涵}

尽管持有不同观点的数学家沿着自己选定的道路前进着, 但最终发现大家不约而同地到达了 同一个地方: 数学研究的对象是一些关系和形式, 这些关系和形式既包含着无限丰富的内容, 又可以用有限符号表征。丰富的数学内容无法简单地归结为逻辑、直觉或形式, 往往融合了 直觉和逻辑, 通过符号的推演构成新的数学内容和体系。身处高科技时代, 各个研究领域都 已进人更深的层次和更广的范畴, 也愈来愈需要数学。数学的语言、演绎、运算和公理化体 系为其他学科的研究提供了具体的和实质的帮助, 甚至曾经一度被认为没有应用价值的抽象 的数学概念与理论, 出人意料地在特定领域中找到了原型和应用, 如数学中的纽结理论与分 子生物学中 DNA 结构的研究有关, 微分几何中的纤维从理论在物理中的规范场中得到应用, 积分几何学的基石拉东变换成为层析成像问题统一的数学基础 [7]。在社会发展中, 数学正在 深人而广泛地渗透到各个领域, 呈现出数学化的趋势, 数学渐渐从科学中脱离出来, 同哲学 一样成为指导其他学科的世界观与方法论。

\section{3. 从教育的视角对数学的诠释}

\section{1. 教育的视角}

国际数学教育委员会主席 Jean Pierre Klahanie 从教育的角度指出 [8]： “没有一门科学像 数学一样, 其学科知识在研究水平上的表述在很大程度上需要转换为用于教学的知识, 并且 这种表述的转换会如此重要; 没有一门学科像数学一样, 其所讲授的内容与其最新研究成果 之间有巨大的距离; 没有一门学科像数学一样, 其所教所学对社会如此重要; 没有一门学科 像数学一样, 具有这样古老的传统, 即研究者同时承担着对教育问题的义务”。从数学科普 读物的编写方面可见一斑, 图书市场上多是铺天盖地的教辅材料, 在份额不多的数学科普读 物中, 大部分还是编译于欧美或韩日的, 或是再版图书, 如再版华罗庚时代知名数学家们写 给中小学生的科普读物, 而当代数学家编写的科普读物少之又少。 


\section{2. 诠释的角度}

数学作为一项教育内容源起于数学学科的产生和发展, 在数学教育从萌芽状态发展到一门专 业, 乃至一门学科的历史进程中大致经历了三个阶段 [9]。第一个阶段由数学家从事数学教学, 学习的载体就是数学家的数学专著, 我国的《九章算术》和西方的《几何原本》都曾做过数 学教科书, 这个阶段数学教育教学的特点是有明确的教学内容, 但很少关注教学方法; 第二 个阶段开始于十九世纪末，伴随着教育的普及和科技的发展，数学在学校教育中开始占有重 要的地位, 很多有识之士提出, “会数学不一定会教数学”, “数学教师是有别于数学家的 另一种职业” ; 第三个阶段以 1908 年国际数学教育委员会的成立为标志, 数学教育开始拥有 专门的研究团队、研究领域、会议和期刊。这三个阶段的划分虽然没有明确的时间分界, 但 可以以从业者的数学教育教学水平为标准, 第一阶段从业者为数学研究者; 第二阶段从业者 不再要求有高深的数学专业知识, 但要懂教育学和心理学; 第三阶段从业者要将数学与教育 心理相互作用。这一渐进的过程也正体现出数学在教育视角下从学术形态向教育形态的转变。 无论在哪个国家和地区, 数学一直以来都是中小学课程体系中所占比重较大的科目。这源于 数学的诸多教育价值 [10], 如准确地把握概念, 解决问题中的方向性思维有助于日后领会工 作的重点; 数学学习中严格、简洁的叙述和论证, 有助于日后谈话和行文简明扼要, 善于经 营, 妥善安排; 如此等等, 不一而足。

3.3. 数学的内涵

张景中院士所提出的教育数学的概念, 认为这个概念有别于数学教育, 他生动地将两者与吃 核桃做比 [11]。核桃是一种有营养的食物, 但很多人不喜欢吃, 主要原因是很难剥皮, 数学 教育即比较容易剥离的核桃, 教育数学则是在不影响核桃品质的前提下改良品种的核桃。也 就是说, 数学教育要靠数学提供素材, 对素材进行教学法的加工使之形成教材; 而教育数学 则是为了教育的需要, 对数学研究成果进行再创造地整理, 这往往需要数学上的创新。近 200 年来, 微积分的教学都从极限概念开始, 但对于大部分初学者, 极限概念很难理解, 于是引 发出这样的思考: 不讲极限可以直接讲导数吗? 即对数学内容进行适当改造, 如今这一探索 已经在我国普通高中各个版本的新课标实验教科书中得以体现。

\section{4. 对数学内涵的再认识}

对大众而言, 数学通常代表着冷酷无情, 鲜有价值和缺少美感, 但事实上, 数学默默地存在 于事物的核心，以某种方式存在于我们所使用和制造的所有事物之中，不但构造了普通的实 物, 还构造了社会、政治、经济当中的种种组织, 甚至成为当代文化的骨架。随着社会的发 展, 开始有越来越多的人意识到从广义的成才角度来看, 一个人接受数学教育的不同层次决 定了所受教育的不同层次。

尽管我们对数学的教育价值有着很高的评价, 但数学课程需要尊重个体的差异, 让不同兴趣 层次, 不同职业追求的学生感受到数学学习的乐趣, 因此课程的设置需要追求动态的数学观, 理性的数学思维, 为所有学习者提供基础的数学课程, 为不同层次的数学学习者提供不同层 次的课程, 提倡多元评价的发展趋势。无论教学的形式如何变化, 教师的数学修养仍然非常 重要, 即通常意义上所说的 “教什么永远比怎么教更重要”。让学生体会到思考的乐趣, 挑 战乐趣, 感受数学带给世界的震撼、力量和解放感, 离不开教师对数学内涵的深入洞察与理 解。

\section{参考文献}

[1] [美] R. 柯朗, $\mathrm{H}$. 罗宾著。左平、张贻慈译。什么是数学 $[\mathrm{M}]$. 上海:复旦大学出版社, 2005 , p.1-3。

[2] Khait, Alexander. the Definition of Mathematics: Philosophical and Pedagogical Aspects[J].Science \& Education, Vol.14(2005), No.2, p.137-159。 
[3] 吴文俊.中国大百科全书.数学[Z].北京:中国大百科全书出版社, 1988，1。

[4] Quirk R. Longman Dictionary of Contemporary English [Z]. London: Longman Group Limited, 1995, p. 376. 黄秦安. 关于数学教育哲学的研究对象与学科特征 $[J]$. 数学教育学报, 2008, 17(6), p.8-11。

[5] 马克思, 恩格斯.马克思恩格斯全集第3卷[M].北京:人民出版社, 1995, p.364.

[6] 黄秦安. 自然 “数学化” 的现象学诠释及其意义追问 [J]. 北京大学学报(哲学社会科学版), 2015, 52(2), p.91-97.

[7] Jean Pierre Klahanie. Did Kuhn Kill Logical Empiricism[J].Philosophy of Science, 1991, 58(2), p.266-267.

[8] 张奠宙.数学教育学导论 $[\mathrm{M}]$.北京:高等教育出版社, 2004, 7p.1-73.

[9] Catherine Lewis, Rebecca Perry. Lesson Study with Mathematical Resources: A Sustainable Model for Locally-led Teacher Professional Learning[J].Mathematics Teacher Education and Development, 2013.16(1), p.3.

[10] 张景中.数学哲学 $[\mathrm{M}]$. 北京:北京师范大学出版社, 2010, p.87-110.

\section{References}

[1] Courant, and Robin. What is Mathematics [M]. Shanghai: Fudan University Press,2005,p.1-3 (in Chinese)

[2] Khait, Alexander. the Definition of Mathematics: Philosophical and Pedagogical Aspects [J].Science \& Education,Vol.14(2005), No.2, p.137-159.

[3] Wenjun Wu. Chinese Encyclopedia of Mathematics [Z]. Beijing: Encyclopedia of China Publishing House, 1988, 1(in Chinese)

[4] Quirk R.Longman Dictionary of Contemporary English [Z].London: Longman Group Limited, 1995 , p. 376.

[5] Qinan Huang. Research Object and Subject Characteristics on Mathematics Education Philosophy[J].Mathematics Education,Vol.17(2008),No.6,p.8-11(in Chinese)

[6] Marx, Engels. Marx and Engels Collected Works [M] .Beijing: People's Publishing House, 1995,p.364 (in Chinese)

[7] Qinan Huang. On the Study Object and Subject Character of Philosophy of Mathematics Education [J]. Journal of Peking University (Philosophy and Social Sciences), Vol.52(2015), No.1,p.91-97 (in Chinese)

[8] Jean Pierre Klahanie. Did Kuhn Kill Logical Empiricism [J].Philosophy of Science, 1991, 58(2), p.266-267.

[9] Dianzhou Zhang.Introduction to Mathematics Education[M].Beijing: Higher Education Press,2004,p.71-73 (in Chinese)

[10] Catherine Lewis, Rebecca Perry.Lesson Study with Mathematical Resources: A Sustainable Model for Locally-led Teacher Professional Learning [J].Mathematics Teacher Education and Development, Vol.16 (2013), No.1, p. 3.

[11] Jingzhong Zhang. The Philosophy of Mathematics [M]. Beijing: Beijing Normal University Press,2010,p.87-110(in Chinese)

作者简介: 田枫 (1977-), 女, 河北山海关人, 课程与教学论 (数学) 专业, 在读博士, 研 
究方向为数学哲学与数学教育. 